\title{
ARQUELOGÍAS ENGENERADAS: LA ODISEA DEL RECHAZO ${ }^{1}$
}

\author{
Daniel Mateos Suárez²
}

\section{Arqueologías Engeneradas: La odisea del rechazo}

Resumen: La interpretación de los hallazgos y restos arqueológicos posee un marcado sesgo androcéntrico contra el que, a partir de la década de 1980, han luchado las denominadas "Arqueologías Engeneradas". Arqueólogas procedentes, en su mayoría, de la academia AngloSajona y Nórdica han reivindicado la importancia de las relaciones de género en la epistemología de la disciplina a pesar del continuo rechazo sufrido por parte del "mainstream". Este trabajo pretende acercarse al debate acerca de la contribución de estas arqueologías a la disciplina atendiendo a su evolución histórica, epistemología y aplicación.

Palabras clave: androcentrismo, arqueologías, engeneradas, epistemología, teoría, museos, genero.

\section{Gendered Archaeologies: An odyssey of rejection}

Abstract:_The interpretation of archaeological findings has a strong androcentric bias which has been challenged by the "Gendered Archaeologies" since the decade of the 1980s. Archaeologists, mainly from the Anglo-Saxon and Nordic Academies have vindicated the relevance of gender relations in the discipline's epistemology despite the constant rejection by the mainstream. This research project aims to take part in the debate on the contribution of these archaeologies to the discipline, by attending to its historical evolution, epistemology and application.

Key words: androcentrism, archaeologies, gendered, epistemology, theory, Museum, gender.

\section{Introducción}

Mis primeros pasos en los estudios de género encontraron en la teoría arqueológica un nuevo espacio de reflexión y teorización y en el que aquellas publicaciones relacionadas con las denominadas como "Arqueologías Engeneradas" captaron mi atención. Sin embargo, pronto descubrí que las más relevantes publicaciones y excavaciones de los últimos años apenas han recogido y reconocido las nuevas perspectivas que dichas arqueologías han proporcionado en las últimas décadas. Esta afirmación pone, teóricamente, en tela de juicio la aportación que estas nuevas líneas han brindado a la disciplina. No obstante, sería interesante analizar si estas han

\footnotetext{
${ }^{1}$ Fecha de recepción: 18/06/2018.

Fecha de aceptación: 05/12/2018.

${ }^{2}$ Becario de Colaboración, Departamento de Historia, Universidad de Oviedo, Oviedo, España; danielmateossuarez@gmail.com.
} 
sido desestimadas por su escasa contribución, o bien, han sido rechazadas por razones políticas. En las siguientes líneas reflexionaremos acerca de cuál ha sido y es la aportación de las "Arqueologías Engeneradas" a la disciplina para, de esta manera, acercarnos a esa incógnita planteada anteriormente.

Antes de sumergirnos en el polémico y controvertible debate en torno a la contribución de estas nuevas perspectivas, consideramos imprescindible realizar una breve reconstrucción del desarrollo histórico que estas han tenido. La influencia de la intitulada como "Segunda Ola del Feminismo" a la Academia trajo consigo una denuncia por parte de arqueólogas americanas y nórdicas acerca del sesgo sexista que poseía la arqueología. Se denunció, por tanto, que la interpretación del pasado "poseía un carácter marcadamente androcéntrico" (Montón 2014: 242) y que la disciplina había sido utilizada como un elemento legitimador de la concepción del género masculino como género dominante. Esta crítica proporcionó a la disciplina nuevas perspectivas que permitieron abordar nuevos temas y reformular otros ya estudiados.

La terminología utilizada para aludir a estas realidades supone una problemática que nos resulta muy difícil abordar. Para ello, hemos decidido acogernos a la definición propuesta por la Encyclopedia of Global Arqueology en la que se acuña el término de "Arqueologías Engeneradas" para designar a todas aquellas arqueologías "que problematizan el sexo, el género y/o la sexualidad en las interpretaciones del pasado y/o de la profesión" (Montón y Meyer 2014: 2372). Bajo este término quedarían enmarcadas los tres tipos de arqueologías que conforman el panorama en la actualidad: Arqueología de Género, Feminista y Queer.

El concepto o análisis de "género" en la arqueología nace como fruto de la concepción de la "diferencia" como el resultado de un proceso sociocultural alejado de aquella concepción naturalista e inevitable. A raíz de este avance empezaron a aparecer diversos estudios que se acercaban al proceso de construcción histórica de estos sistemas de género, así como aspectos tan específicos como la simbología, los roles o la ideología de género. En 1984 Margaret W. Conkey y Janet D. Spector publicaron un artículo que "inauguró" la "Arqueología de Género". En esta publicación criticaron y denunciaron el carácter androcéntrico que caracteriza a la disciplina y formularon un nuevo discurso arqueológico en el que la categoría de género adquiere una importancia 
capital. Definen el "género" como el resultado de una construcción social en la que históricamente los dos sexos han negociado y que está en constante evolución y renegociación. Por tanto, el concepto de género emana de los diversos cambios y contextos que se dan en un espacio y tiempo concreto y su estudio "requiere una investigación de los procesos históricos que los determinan” (Sanahuja 2002: 77).

Este nuevo campo de investigación gozó de cierto "éxito" tal y como certifican las diversas y numerosas encuentros y congresos académicos que desde la década de 1980 se han celebrado. La primera reunión de esta índole en España tuvo lugar en Santiago de Compostela, en el año 1992 (Montón 2014: 243). En el ámbito internacional debemos destacar los encuentros celebrados en Estados Unidos, en los que muchas mujeres encontraron referentes que habían vivido experiencias similares. Además, de los numerosos debates planteados surgió una de las obras más representativas y relevantes de estas nuevas perspectivas: Engendering Archeology, escrita por Joan Gero y Margaret Conkey en el año 1991 (2014: 243).

La arribada de la denominada como "Tercera Ola de Feminismo" fue progresivamente incorporando nuevas inquietudes y problemáticas. Fundamentalmente, proporcionó una nueva manera de entender la relación entre el sexo y el género, así como una reformulación de aquellos aspectos vinculados con la identidad personal. A la luz de estas nuevas prerrogativas nace, a finales de los años 90, y se desarrolla la conocida como "Arqueología Queer" que busca romper con el "modelo hegemónico de la heterosexualidad" (244). Esta nueva configuración arqueológica planteaba que el sexo también era el resultado de una construcción sociocultural. Estos nuevos postulados no obtuvieron gran aceptación entre los arqueólogos puesto que la mayoría siguieron diferenciando en sus interpretaciones entre sexo y género.

Esta escasa aceptación se debe en gran parte al desafío de esta arqueología a la naturaleza cronocéntrica de la disciplina, pues consideraban que "el cronocentrismo es el falocentrismo de la arqueología" (Sanahuya 2002: 84). No obstante, no podemos dejar en el olvido la importante repercusión que han tenido los "llamados terceros géneros y los análisis de interseccionalidad entre el género y otras adscripciones sociales" (Montón Subías 2014: 243). En esta misma línea, podemos observar cómo, en 
los últimos años, se han incorporado análisis que se acercan al proceso de construcción de la identidad personal.

Debemos reseñar que esta corriente arqueológica, que no ha gozado de un gran éxito en el territorio nacional, "ha revelado el carácter heteronormativista imperante en las ciencias sociales" (Moral 2014: 249). Este sesgo heteronormativista que persigue unos intereses concretos ha estado y aún sigue estando presente en la epistemología arqueológica mainstream. El objetivo de esta perspectiva es, por tanto, generar nuevas formas de conocimiento que se opongan al "contenido de la producción científica hegemónica, a la norma y a los procedimientos" (Montón y Lozano 2012: 164). En definitiva, esta nueva configuración surge como una oposición a la norma, es decir, tiene el objetivo de deconstruir el discurso normativo.

Las "Arqueologías Engeneradas" proporcionan perspectivas eminentemente teóricas. Este carácter teórico podría explicar que España no fuera pionera en la formulación de estas nuevas perspectivas y es que, podemos afirmar que no será hasta finales de la década de 1980 cuando los arqueólogos españoles comiencen a reflexionar acerca de la historia de la disciplina, su situación y su futuro (Oria Segura 1999: 10). Este retraso en comparación con el mundo anglosajón y/o nórdico, fomentado especialmente por el aislamiento cultural que el país sufrió durante la dictadura, explicaría el "tardío" desarrollo de las "Arqueologías Engeneradas" en el territorio nacional. La utilización de la disciplina por parte del aparato franquista reforzaría aún más ese carácter androcéntrico que ha caracterizado a la arqueología. Esta particularidad, así como la desconexión académica y cultural, podría explicar el poso androcéntrico que aun caracteriza el desarrollo de la disciplina en España.

Sin embargo, en los últimos años estamos asistiendo a una autentica renovación teórica de la disciplina, que, en el ámbito clásico, concretamente adscrita a la vertiente Post-Colonialista, vemos en investigadores como Alicia Jiménez y/o Jaime Vives Ferrándiz (Machuca 2014: 44). No obstante, cabe reseñar qué si bien es verdad que España no participó en el primer desarrollo de las “Arqueologías Engeneradas", la implantación de estas en la academia constituye toda una excepción en el ámbito mediterráneo, pues desde su llegada han gozado y siguen disfrutando de cierto éxito (Diaz-Andreu y Montón-Subías 2013: 440). 
La arribada de estas nuevas perspectivas arqueológicas al territorio nacional tuvo lugar durante los primeros años de la democracia y el desarrollo en España de la denominada "Segunda Ola del Feminismo", lo que, junto al gran número de vacantes en las universidades, explica el rapido y solido desarrollo que estas tuvieron. Esta implantación se ejemplifica en la presentación de estas "Arqueologías Engeneradas" en las "II Jornadas Catalanas de la Dona" por parte de Maria Encarna Sanahuja (2013: 441). Actualmente podemos afirmar que estas nuevas perspectivas han consolidado en las últimas décadas su posición en el ámbito nacional tal y como ilustran las numerosas conferencias y publicaciones al respecto. Sin ánimo de ignorar a muchos autores o autoras, podríamos citar a investigadores e investigadoras como Eva Alarcón García, Margarita Sánchez Romero (2015), Sandra Montón Subías (2014), Gemma Lugo Espinosa o María del Pilar Alberti Manzanares, entre otros y otras. Sin embargo, al igual que sucede en el ámbito anglosajón, no existe una verdadera oposición al mainstream que aún sigue imperando.

\section{El marco procesualista y su continuación}

La pronta incursión nórdica en las perspectivas arqueológicas de género, no solo suscitan un debate en torno a la "inauguración" de las "Arqueologías Engeneradas", sino que retrasarían la aparición de estas al marco procesual. Consideramos, por tanto, imprescindible hacer referencia a esta "Nueva Arqueología" en tanto que precedente del Post-Procesualismo y perpetuadora del sesgo androcéntrico. En las siguientes líneas trataremos de analizar en clave de género esta escuela anglosajona como antesala al post-procesualismo y como antecedente del desarrollo en la década de 1980 de las “Arqueologías Engeneradas".

El nacimiento del procesualismo en la década de 1960 está caracterizado por la democratización masculina de la disciplina. Esta nueva perspectiva que nace en el seno de la Universidad de Cambridge posee un sesgo claramente androcéntrico y sus fundadores y más importantes teóricos son en su inmensa mayoría hombres. En las siguientes líneas trataremos de responder la siguiente pregunta: ¿Donde estaban las 
mujeres? Esta tendencia sigue vigente en la actualidad tal y como advertimos en las principales conferencias donde seguimos observando una presencia mayoritariamente masculina y en las principales universidades europeas, donde las "Arqueologías Engeneradas" siguen sin estar presentes de forma generalizada en las diversas agendas académicas; en Inglaterra solamente un $9 \%$ de los profesores de universidad de arqueología son mujeres (Pope 2011: 61).

La arqueología procesual trajo consigo, tal y como enunciamos anteriormente, un proceso democratizador en el que muchos estudiantes procedentes de la clase trabajadora fueron admitidos en las principales universidades. Esta supuesta apertura de la disciplina solo se limitó, en su gran mayoría a los hombres suponiendo, de esta manera, una aparente regresión de la presencia de la mujer en comparación con los datos presentados en las últimas investigaciones y que parecen destacar una importante presencia femenina en la disciplina durante la década de 1920 y 1930 (2011: 63-64). Sin embargo, este retraso no implicó que las mujeres no tuvieran influencia en esta nueva perspectiva y es que grandes nombres como el de Sally Binford tuvieron un importante peso en el origen y posterior desarrollo de la "Nueva Arqueología".

En las décadas de 1960 y 1970 advertimos un ligerísimo y apenas destacable incremento en los porcentajes de mujeres cursando estudios arqueológicos. Sin embargo, estas cifras esconden los problemas que las mujeres tenían que enfrentar cuando se casaban y que les acababa obligando a aparcar su carrera profesional para asumir un rol materno. En este contexto también observamos el fenómeno del rol de la "mujer del arqueólogo" y es que muchas de las mujeres que siguieron vinculadas a la disciplina adoptaron un rol de apoyo a la carrera de sus maridos. Un caso muy significativo es el de Helen Wace que trabajó junto con su marido en Micenas (77). Generalmente, estas mujeres siguieron la estela de sus maridos, aunque algunas tuvieron la posibilidad de excavar y llevar a cabo sus propios proyectos.

En definitiva, si bien es verdad que la influencia de la arqueología procesual propició una democratización mayoritariamente "masculina" de la disciplina, esta supuso un pequeño avance que hizo posible el ingreso de muchas mujeres en las principales instituciones académicas. Además, en los epígrafes pretéritos hemos comprobado que las mujeres siempre han estado presentes en los diversos procesos y 
evoluciones de las perspectivas arqueológicas y que ha sido la visibilidad de estas lo que realmente ha ido cambiando. A modo de síntesis, la New Archaeology se erige como un pretexto del origen de las "Arqueologías Engeneradas" en la que paralelamente se intensifica el sesgo androcéntrico y la mujer, aunque parezca paradójico, va progresivamente integrándose en los principales resortes del mainstream, desde los que después articulará su crítica.

\section{Epistemología feminista}

La exposición de las nuevas perspectivas arqueológicas previamente presentadas desde un punto de vista epistemológico resulta imprescindible para poder abordar el dualismo o la relación entre las "Arqueologías Engeneradas" y el "Feminismo". Para afrontar esta problemática seguiremos las teorías expuestas Sandra Harding (1986), en las que afirma que estas nuevas líneas teóricas han seguido en su desarrollo los mismos pasos que el feminismo, es decir, un primer feminismo empírico, un posterior feminismo "Standpoint" y un último feminismo Post-Modernista. Este análisis debe ser enmarcado en su contexto geográfico, pues existe una enorme diferencia en cuanto a desarrollo entre el ámbito noruego, el Anglo-Sajón y el mediterráneo (Lozano Rubio 2011: 22)

Las epistemologías feministas han proporcionado nuevas estrategias en el proceso científico y han formulado nuevas visiones en relación con la objetividad y la justificación del conocimiento. Reclaman, desde presupuestos críticos, que la ciencia adquiera un carácter más realista y libre del sesgo androcéntrico. El primero de ellos, el conocido como feminismo empírico, analiza como los/las feministas llevan a cabo el proceso científico y como argumentan, desde presupuestos empíricos, que el método científico esconde claros matices androcéntricos. Según este feminismo empírico, el problema radicaría en el uso del método (2011: 24), es decir, reclama una ciencia libre de influencias erróneas.

Las primeras arqueólogas que empezaron a articular perspectivas de género estaban adscritas a la corriente procesualista, tal y como hemos demostrado en el 
apartado anterior. Esta "Nueva Arqueología" se vendría a corresponder con esa primera epistemología feminista, es decir, la empírica. Esta epistemología planteó un nuevo modelo en el que los grupos humanos operaban dentro de un sistema complejo con el fin de llegar a un equilibrio (25). Estas feministas empíricas empezaron a reclamar la importancia del contexto de los hallazgos y emprendieron una búsqueda de la "mujer". La crítica academicista pronto rechazó estos análisis pues desde dicha crítica argumentaban que carecían de fuentes empíricas y que la atribución de determinados objetos a un género especifico planteaba una enorme dificultad.

La reacción de dichas arqueólogas, especialmente en el ámbito noruego, se centralizó en el estudio de los contextos funerarios. En el año 1976, Liv Helga Dommasnes publicó la primera tesis de "Arqueología Engenerada" en Escandinavia, en la que analizó la división sexual del trabajo en los últimos años de la Edad del Hierro en Noruega siguiendo los métodos y teorías procesalistas (26). Esta publicación fue todo un éxito y consolidó el feminismo empírico como una corriente que posibilitaba el contacto con otras corrientes no feministas y que, sin duda, colocaba a las denuncias feministas en un importante plano académico. Por tanto, podemos afirmar que muchas arqueólogas como Elisabeth Brumfiel han contribuido de manera relevante a la corriente procesualista, sin embargo, no sería del todo correcto identificar el feminismo empírico con el procesualismo, pues si bien es verdad que guardan muchas similitudes, existen algunas diferencias, especialmente en relación con los valores epistemológicos.

Estos primeros intentos no fueron totalmente eficaces pues aún subsistían numerosos matices androcéntricos en la disciplina. A raíz de esto, surgen en la década de 1980 las teorías que denuncian la existencia de ciertos grupos sociales que reciben un privilegio epistemológico debido a la marginada localización social en la que habitan (Ibid: 28). Argumentaban que aquellas personas en situaciones privilegiadas tendían a ser menos críticas y por dicha razón, menos objetivas. Por el contrario, aquellas personas en situaciones más complicadas estaban motivadas a entender cuál eran las razones de sus problemas, lo que les permite estudiar profundamente la realidad y revelar los diversos problemas y tensiones que en esta actúan.

Estos presupuestos emanan originalmente de las ideas marxistas acerca de la posición epistemológica del proletariado. Sin embargo, esta corriente adopta esas ideas 
y presenta a la mujer como aquel grupo social oprimido por la sociedad patriarcal. Centran su atención en analizar como las estructuras de género moldean a las sociedades jerárquicamente, algo que los hombres no perciben. Por tanto, estas teorías, enmarcadas en la denominada Standpoint Theory, disfrutarían de un privilegio epistemológico pues se basan en el análisis social por parte de mujeres que son conscientes de que ocupan un lugar inferior impuesto por las estructuras de género. Esta teoría se ha ido complejizando pues autoras como Sandra Harding han reflexionado acerca de la diversidad entre las mujeres y aspectos tales como la raza, el origen étnico o la sexualidad.

A comienzos de la década de 1990 se instaura en muchas arqueólogas y autoras una visión pesimista. Estas corrientes denominadas "Post-Modernistas" reaccionan ante aquellos valores ilustrados que conformaron la modernidad (32). Esta epistemología reclamaba la descentralización del sujeto y el carácter parcial de todo conocimiento (32). Argumentaban que nadie es puramente objetivo y a consecuencia, el conocimiento puede ser utilizado como una herramienta de poder (opresivo o no). Esta última corriente cerraría esta división epistemológica que presentó Sandra Harding y que, sin duda, tiene unos límites muy difusos, pues muchas autoras empíricas evolucionaron hacia ideas propias de la Standpoint Theory, así como muchas de esta última influenciaron la corriente Post-Modernista. Estas tres epistemologías han interactuado interseccionalmente dentro de los dos grandes marcos teóricos de la arqueología: El procesualismo y el Post-Procesualismo.

\section{Museos y género}

Los hallazgos arqueológicos y las interpretaciones derivadas de estos son presentados ante la sociedad en museos. Muchos académicos reclaman que el patrimonio cultural conservado en dichos espacios debe ser sometido a una revisión en clave de género. Las reconstrucciones históricas que presentan los museos "son tomadas como ciertas por el público dado el contexto oficial en el que se encuentran" (DíazAndreu 2005: 27). Sin embargo, esta reconstrucción no ha sido realizada en igualdad y 
por dicha razón, son muchas las iniciativas que se están articulando en torno a esta denuncia. Un ejemplo es el destacado proyecto puesto en marcha en el año 2011 por la Subdirección General de Museos Estatales y que recibe el nombre de "Patrimonio en Femenino", al que le siguió un año después el proyecto "Descubrimiento de las Ausencia y Silencios” (Hernández 2015: 16). Estas campañas reivindican el papel activo y dinámico que las mujeres han tenido en los museos y denuncian aquellos comportamientos exclusivos que han caracterizado a diversos órganos de gobiernos e instituciones patrimoniales.

Estas reivindaicones proponen una reformulación de la museología en la que se incluya la perspectiva género y en la que se valore a la mujer teniendo en cuenta su especificidad con las mismas condiciones que la de los hombres. Por tanto, plantean la eliminación de todo sesgo androcéntrico y contenido patriarcal. En resumen, exigen que se "cuiden los discursos monográficos, resaltando las diferentes identidades como un nuevo elemento a tener en cuenta" y destacando la igualdad del hombre y de la mujer como un aspecto imprescindible (2015: 16). Esta corriente no asevera en ningún momento que mujeres y hombres hayan realizado las mismas actividades, sino que, denuncian el que se le dé más importancia a la actividad del hombre.

En el ámbito nacional debemos destacar las aportaciones de María Ángeles Querol y Francisca Hornos. Estas autoras han realizado un exhaustivo análisis del Museo Arqueológico Nacional con el fin de demostrar la carencia de objetividad y neutralidad de las exposiciones, así como de las representaciones históricas. Este tipo de estudios han centrado su atención en las representaciones de las personas, es decir, que trabajos realizan, en que postura están, qué lugar ocupan en la escena o que vestimenta portan. En 2011 las expertas previamente citadas publicaron cinco estudios en los que analizaban cinco de los principales museos arqueológicos del territorio nacional.

Realizaron un análisis comparativo en el que analizaron varios aspectos como, por ejemplo, los porcentajes de mujeres representadas en los cinco museos. La media de los cinco apenas supera el 23\%, lo que sin duda demuestra una desigualdad. Está se acentúa cuando observamos que no aparecen en ningún ambiente productivo. En definitiva, estos porcentajes nos evidencian un discurso marcado por "el protagonismo masculino y la importancia de las actividades propias de hombres" (Querol 2014: 275). A modo de 
síntesis, los museos son auténticos irradiadores de mensajes e influencias hacía la sociedad, y, por tanto, deben cuidar sus discursos y demostrar una actitud mucho más responsable y comprometida con el fin de alejar a la sociedad de esa idea de "que la invisibilidad, inferioridad y la escasa importancia de las mujeres y sus trabajos ha sido siempre igual" (2014: 279).

\section{Arqueologías Engeneradas en el proceso de reconstrucción e interpretación}

Tras este pequeño análisis de las “Arqueologías Engeneradas” y su contribución a la disciplina, queremos presentar sus aproximaciones teóricas aplicadas al mundo clásico con el fin de acercarnos a su planteamiento practico. En primer lugar, debemos subrayar que la mayoría de trabajos al respecto proceden del campo de la arqueología prehistórica. Sin embargo, en los últimos años se están publicando trabajos adscritos tanto al mundo clásico como al periodo medieval. Con este epígrafe pretendemos alejarnos en cierta medida del carácter eminentemente teórico por el que son criticadas estas perspectivas y en las próximas líneas presentaremos de forma muy reducida una investigación vinculada al mundo antiguo en el que las Arqueologías Engeneradas han dibujado nuevas líneas de interpretación e investigación.

Entre las numerosas investigaciones llevadas a cabo en los últimos años, la comparación que Ana Delgado y Meritxell Ferrer (2011) realizaron de dos asentamientos fenicios, en el sur de la península iberica y en el oeste de Sicilia, constituye un gran ejemplo de cómo los hallazgos materiales de los espacios domésticos y funerarios pueden revelar aspectos fundamentales de la convivencia y de la diferencia de las identidades hegemónicas de género. Este acercamiento a la esfera doméstica ha comprobado un aspecto fundamental, es decir, la coexistencia de personas de diverso origen cultural y geográfico en ambos ámbitos coloniales.

Por su parte, el análisis del ajuar hallado en el espacio funerario evidencia la participación de estos elementos ajuarísticos en la construcción de las identidades y las relaciones de género. Concretamente, la presencia de objetos relacionados con la comida y el cuidado del cuerpo aparecen, generalmente, asociados a los restos 
femeninos, lo que, siguiendo la tesis de que estos actúan como una extensión de la persona, reflejarían el rol de las mujeres tanto en esa sociedad como en el discurso colonial.

En definitiva, la investigación presentada en las líneas pretéritas nos demuestra de forma fehaciente como un análisis en clave de género nos permite acercarnos a un estudio y análisis de la sociedad de las colonias fenicias. Es decir, desde los presupuestos teóricos de las “Arqueologías Engeneradas”, se ha podido reconstruir, no solo las diversas relaciones de género, sino el contexto social de este ámbito colonial. Por tanto, podemos determinar que estas nuevas líneas contribuyen de manera decisiva en el proceso de interpretación y reconstrucción de los periodos históricos desde todos los puntos de vista, no únicamente desde el de las relaciones de género.

\section{Conclusiones}

En los epígrafes anteriores hemos analizado cuales han sido las diferentes aportaciones teóricas y metodológicas a la disciplina. De este mismo modo, también debemos reseñar su contribución a la práctica profesional de la Arqueología. Dejando a un lado esta inestimable aportación, querríamos subrayar, de manera especial, la dimensión humanista que la perspectiva de género le ha otorgado a la disciplina. Estas reformulaciones y nuevas perspectivas han permitido a la teoría arqueológica profundizar en temas relacionados con la vida cotidiana y la agencia humana. Esto ha supuesto una autentica renovación en el análisis de los espacios cotidianos resaltando su importancia en el proceso de reconstrucción histórica. Además, esta dimensión humanista permite analizar y estudiar las relaciones humanas, lo que, sin duda, proporciona una información de gran importancia en dicho proceso.

Un aspecto que ejemplifica la importancia que estas nuevas perspectivas han cobrado, así como el interes que han suscitado, es el considerable volumen de publicaciones y la constante dialéctica y evolución de sus principios teóricos. No obstante, debemos mencionar y valorar negativamente el estrecho vínculo que estas guardan con el espectro político, pues tanto las arqueologías feministas como las de 
queer se han postulado como auténticas armas contra el patriarcado, es decir, tienen un marcado carácter revolucionario que hace que se las vincule con otras perspectivas arqueológicas como la Post-Colonial o la indígena. En esta misma línea, otro aspecto que cabría "criticar" es su vinculación con el feminismo como movimiento, pues, si bien es verdad, que nace gracias a este, muchas autoras están intentando alejarse de esta vinculación; sin embargo, la mayoría de los trabajos siguen mostrando ese compromiso con el feminismo. Consideramos que esta vinculación politica podría afectar negativamente al desarrollo de esta nueva perspectiva, es decir, debe entenderse como un nuevo enfoque de una disciplina científica y, por ende, su carácter debe ser científico y no esencialmente político.

Sin embargo, esta separación del movimiento feminista, que desde nuestro punto de vista no puede ser demasiado amplia, no debe, en ningún caso, permitir que el mainstream transforme la arqueología de género ni elimine el compromiso de los autores y las autoras. Esta separación no debe implicar la eliminación del compromiso y el objetivo de acabar con el patriarcado; estas actitudes u objetivos deben convivir con la integración de este enfoque en una disciplina cuyo objetivo es y debe ser reconstruir el pasado de forma fidedigna. En resumen, la arqueología de genero debe, desde nuestro punto de vista, desembarazarse de su vinculación con el movimiento feminista sin, aunque parezca paradójico, alejarse totalmente de él, para modificar completamente la disciplina e integrar una perspectiva científica cuyo objetivo no debe ser otro que reescribir la historia de forma correcta, lo que implica, tal y como han demostrado los autores y las autoras que hemos reseñado a lo largo de esta contribución, acabar con el sesgo androcéntrico y restructurar la historia en términos igualitarios, lo que a su vez, y con esta última relación pretendo cerrar esta reflexión, coincide con el principio fundamental del feminismo, la igualdad.

Tras este pequeño inciso, debemos destacar muy positivamente que estas perspectivas rescatan del olvido aspectos tan relevantes como la reproducción, el mantenimiento del ajuar doméstico o el procesado de alimentos. En definitiva, el sesgo androcéntrico que ha caracterizado históricamente y aún sigue caracterizando la disciplina, ha pasado por alto aspectos básicos para el funcionamiento de cualquier sociedad o grupo. Los arqueólogos han interpretado el pasado como un "precedente lógico del presente" (Sanahuja 2002: 64) en el que las mujeres siempre han trascendido 
en la esfera doméstica y bajo la estructura de los "valores de la familia occidental moderna" (2002: 65). En ningún caso se considera la posibilidad de que pudieran haber existido comportamientos y relaciones diferentes y/o alternativas a las actuales entre sexos.

Asimismo, es relevante señalar que esta arqueología no pretende únicamente estudiar y analizar aquello que realizaban las mujeres, sino conocer también que hacían los hombres. Es decir, busca alejarse de una interpretación del pasado hecha desde los estereotipos propios de la sociedad contemporánea. Por tanto, las "Arqueologías Engeneradas" no pretenden recrear una historia en la que las mujeres sean las protagonistas, sino conocer e interpretar el pasado de manera fidedigna mediante la reivindicación de la "mujer" en aquellas categorías en la que no ha sido incluida y desterrar los paradigmas androcéntricos que han imperado en la disciplina. A pesar de los numerosos y dificultosos éxitos alcanzados, a un nivel "macro", las "Arqueologías Engeneradas" aún siguen intentando transformar los presupuestos teóricos de la disciplina mediante la comprensión de la función del género en tanto que relaciones, roles o ideología.

Desde este artículo queremos denunciar el continuo rechazo que aun hoy siguen sufriendo estas "Arqueologías Engeneradas". En los epígrafes anteriores hemos demostrado que estos nuevos presupuestos han aportado un soplo de aire fresco a la actividad arqueológica y una perspectiva que la acerca hacía una representación fidedigna y correcta del pasado. La antigua y, desafortunadamente, aún muy presente actitud negativa del arqueólogo hacía la teoría ha perdido toda su vigencia por lo que, no tener en consideración estas cuestiones acerca de la subordinación, la desigualdad o el género, es una mera elección teórica que tiene sus consecuencias ideológicas, políticas, sociales y, por supuesto, científicas. Es fundamental que se reinterpreten trabajos anteriores y, aún más importante, desde nuestro punto de vista, que se enseñe, a las nuevas generaciones, a escribir correctamente la historia, una historia verdadera, una historia igualitaria y no androcéntrica. Es imprescindible que se deseche la visión pasiva de la mujer durante la historia y que se visibilice, tal y como han demostrado autores como Alfonso Monsalve Romera, Margarita Sánchez Romero y A. González Martín (2015), que las mujeres desempeñaban, en las sociedades prehistóricas y, si nos apoyamos de nuevo en el interesantísimo trabajo de Ana Delgado y Meritxell Ferrer 
(2011), también antiguas, un rol crucial. En definitiva, un discurso histórico igualitario podría emplearse como una herramienta muy eficaz, a traves de su proyección en las diferentes etapas educativas y en los museos, para "confeccionar" una sociedad justa e igualitaria.

Sin embargo, consideramos capital, para el desarrollo de dichas perspectivas arqueológicas, que estas se articulen en torno a la disciplina arqueológica como elementos adyacentes que permitan reconstruir e interpretar el pasado de la manera más correcta posible. Es decir, estas nuevas líneas no pueden desvincularse de la Arqueología como disciplina y funcionar como corrientes independientes pues, tanto las diferentes epistemologías como los diversos métodos de análisis, deben converger en un mismo objetivo: una interpretación y reconstrucción del pasado fidedigna, apolítica y libre de presupuestos androcéntricos propios del mainstream.

\section{Referencias bibliográficas}

Alarcón, Eva y Margarita Sánchez. 2015. "Arqueología feminista de las mujeres y del género en la prehistoria de Andalucía”. Menga, 6: 33-60.

Cinta, Marta. 2012. "Género y Arqueología: un esquema de la cuestión”. Estrat Critic, 6: 177-187.

Clinger, J. 2005. Our Elder. Six bay area life stories. Londres: Bloomington.

Delgado, Ana y Meritxell Ferrer. 2011. "Life and death in Ancient Colonies. Domesticity, Material Culture, and sexual Politics in the western phoenician World, eight to sixth centuries". The archaeology of Colonialism: Intimate encounter and sexual effects. Eds. Barbara Voss y Eleanor Casella. Cambridge: Cambridge University Press. 195-214.

Díaz-Andreu, Margarita. 2005. "Género y Arqueología: una nueva síntesis". Arqueología y Género. Ed. Margarita Sánchez. Granada: Universidad de Granada. 13-51.

Díaz-Andreu, Margarita y Sandra Montón-Subías. 2013. "Gender and Feminism in the Prehistoric Archaeology of Southwest Europe". A companion to Gender Prehistory. Ed. Diana Bolger. Oxford: Wiley-Blackwell. 438-457

Geller, Pamela. 2009. "Identity and difference: Complicating Gender in Archaoelogy". Annual Review of Anthropology, 38: 65-81. 
Harding, Sandra. 1986. The Science Question in Feminism. New York: Cornell University Press.

Hernández, Francisca. 2015. "Introducción. La Museología: Entre la tradición y la posmodernidad". Complutum, 26 (2): 9-26.

Lozano, Sandra. 2011. "Gender Thinking in the Making: Feminist Epistemology and Gender Archaeology". Norwegian Archaeological Review, 44 (1): 21-39.

Machuca, F. 2014. "Viejos problemas, nuevos enfoques: las aportaciones de la teoría poscolonial al estudio de la antigüedad". Revista Historia Autonoma, 4: 33-46.

Monsalve, Alfonso; Margarita Sánchez y A. González. 2015. Un análisis preliminar desde la arqueología y la antropología física: Las comunidades del Bronce de la Mancha: el caso del Cerro de La Encantada. Menga, 5: 175-177.

Montón, Sandra. 2014. “Arqueologías Engeneradas. Breve introducción a los estudios de género en Arqueología hasta la actualidad”. ArqueoWeb, 15: 242-247.

Montón, Sandra y Sandra Lozano. 2012. "La arqueología feminista en la normatividad académica”. Complutum, 23 (2): 163-176.

Montón-Subías, Sandra y Will Meyer. 2014. "Engendered Archaeologies". Encyclopedia of Global Archaeology. Ed. Claire Smith. New York: Springer. 2372-2381.

Moral, Enrique. 2014. “¿Es el sexo al género lo que la naturaleza a la cultura? Una aproximación queer para el análisis arqueológico". ArqueoWeb, 15: 248-269

Oria, M. 1999. "El estado de la arqueología clásica en España: Propuestas para un debate necesario". SPAL, 8: 9-19

Pope, Rachel. 2011. "Processual archaeology and gender politic. The loss of inocence". Archaeological dialogues, 18: 58-86.

Querol, María Ángeles. 2014. "Museos y mujeres: la desigualdad en Arqueología". ArqueoWeb, 15: 270-280.

Sanahuja, María Encarna. 2002. Cuerpos sexuados, objetos y prehistoria. Madrid: Catedra. 